\title{
Avaliação das alterações de oxigênio dissolvido e temperatura no Arroio Esperança
}

\author{
Fernando Ernesto Ucker, Anna Paula Ferreira Batista Goldfeld Ucker, Pedro Daniel da Cunha Kemerich, \\ Marcelo Tsuyoshi Haraguchi, Felipe Corrêa Veloso Santos, Wilian Fernando Borba \\ Laboratório de Planejamento e Monitoramento Ambiental - LPMA/UFSM \\ Curso de Engenharia Ambiental da Universidade Federal de Santa Maria - UFSM/CESNORS
}

\section{Resumo}

Situado do município de Santa Maria - RS, o Arroio Esperança encontra-se em uma microbacia com situações de urbanização tanto regular quanto irregular. Através das degradações causadas pelo homem, a qualidade dessa água têm sido alterada constantemente. Este trabalho teve como objetivo realizar a avaliação das alterações de oxigênio dissolvido e da temperatura deste Arroio. A pesquisa foi realizada entre os meses de março e julho de 2011, onde foram avaliados, in situ, as variáveis oxigênio dissolvido e temperatura da água. Após avaliação, a água do Arroio Esperança em pouco pode ser classificada de acordo com a Resolução 357/2005 do Conselho Nacional do Meio Ambiente.

Palavras-chave: Arroio Esperança; Oxigênio Dissolvido; Microbacia; Degradação.

\begin{abstract}
Located in the municipality of Santa Maria - RS, the Arroio Esperança is a small catchment situations urbanization regular and irregular. Through the degradations caused by man, the quality of this water has been changed constantly. This study aimed to carry out the assessment of changes in dissolved oxygen and temperature of this Arroio. The survey was conducted between March and July 2011, were evaluated in situ, the variables dissolved oxygen and water temperature. After assessment, the water in the Arroio Esperança some can be classified according to Resolution 357/2005 of the National Council of the Environment.
\end{abstract}

Keywords: Arroio Esperança; Dissolved Oxygen; Watershed; degradation. 


\section{INTRODUÇÃO}

Substância vital para o desenvolvimento dos ecossistemas, a água faz parte do patrimônio do nosso planeta. De acordo com Telles e Costa (2007), podemos considerar que a quantidade total de água na terra é de 1.386 milhões de $\mathrm{km}^{3}$, onde apenas $2,5 \%$ deste volume constituem-se de água doce. No Brasil, a região coberta por água doce chega a ocupar aproximadamente 55.457 $\mathrm{km}^{2}$, o que equivale a $8 \%$ da reserva de água doce do planeta, destacando assim nosso país no cenário mundial como um território de vasta rede hidrográfica.

Entretanto, o crescimento desordenado da população mundial, juntamente com o uso indevido da água, está levando a contaminação de mananciais, tornando a cada dia a água como um bem ainda mais valioso. O intenso uso e exploração dos recursos hídricos, já limitados, nas atividades de produção e consumo estão degradando-os. Diante disso, é cada dia mais visível a necessidade de se reduzir a poluição hídrica.

O conceito qualitativo da água é muito mais abrangente do que a caracterização da água pela fórmula $\mathrm{H}_{2} \mathrm{O}$. A água pura é um líquido incolor, inodoro, insípido e transparente. Porém, nunca é encontrada no seu estado de absoluta pureza, por ser um solvente universal, contendo várias impurezas como algas, areia, argilas, minerais e compostos orgânicos. Esta ótima solubilidade é aumentada pela solubilização de dióxido de carbono existente na atmosfera e no solo como resultado da decomposição de matéria orgânica (SPERLING, 1996). A qualidade da água não se refere ao grau de pureza absoluto ou próximo deste, mas sim a um padrão mais próximo possível do natural da água, ou seja, como ela se encontra nas nascentes, antes do contato do homem (BRANCO, 1991). O termo "qualidade de água" se trata das características físico-químicas e biológicas e, dependendo destas características, são determinados diversos destinos para a água (MERTEN; MINELLA, 2002).

A qualidade da água de uma microbacia pode ser influenciada por diversos fatores como o clima, a cobertura vegetal, a topografia, a geologia, bem como o tipo, o uso e o manejo do solo da bacia hidrográfica (PEREIRA, 1997). Os principais tipos de poluição aquática são a orgânica, microbiana e a inorgânica. A poluição orgânica é caracterizada pela presença de matéria orgânica na água, assim quanto maior a quantidade de matéria orgânica disponível, maior será a população de organismos que a decompõem, portanto maior será a quantidade de oxigênio consumida (NUVOLARI, 2003). A poluição microbiana é resultante da descarga de resíduos humanos que possui grande variedade de patógenos, entre eles bactérias, vírus, protozoários ou organismos multicelulares, que podem causar doenças gastrointestinais (TUNDISI, 2005).

Diversos estudos mostram que as variações do oxigênio dissolvido levaram algumas espécies de peixes a desenvolverem adaptações para suportar as condições periódicas de hipóxia (ALMEIDA-VAL et al. 1993; SOARES, 1993; ALMEIDA-VAL e HOCHACHKA, 1995). Já os limites de temperatura, segundo Sánchez-Botero (2000), podem variar de região para região, porém, geralmente encontram-se na faixa de $20^{\circ} \mathrm{C}$ a $34^{\circ} \mathrm{C}$. Estes valores, segundo o mesmo autor, são próximos as temperaturas letais para uma grande quantidade de peixes de água doce. Pelo exposto anteriormente, objetivou-se neste trabalho a avaliação da mudança do oxigênio dissolvido e da temperatura da água no Arroio Esperança.

\section{MATERIAL E MÉTODOS}

O trabalho foi realizado em uma microbacia com característica altamente urbanizada (Figura 1), envolvendo situações de urbanização regular e irregular, que engloba parte da área central do município de Santa Maria - RS, cobrindo uma área de aproximadamente 44 hectares. A coleta de amostras de efluente foi realizada entre os meses de março e julho de 2011, no Arroio Esperança. Realizou-se uma coleta por semana, sem precipitação nas últimas 24 horas. A avaliação era realizada in situ.

As amostras de água foram coletadas em quatro pontos pré-definidos cujas coordenadas são: Ponto $1\left(29^{\circ} 40^{\prime} 35.507^{\prime \prime}\right.$ S e $53^{\circ} 48^{\prime} 58.717^{\prime \prime}$ W), Ponto $2\left(29^{\circ} 40^{\prime} 39.168^{\prime \prime}\right.$ S e $\left.53^{\circ} 48^{\prime} 54.450^{\prime \prime} \mathrm{W}\right)$,

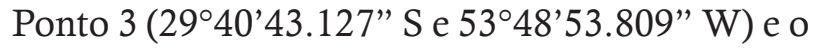

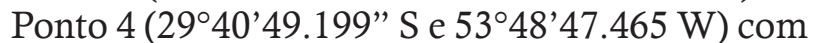
altitude entre 96,84 e 102,4 m, conforme figura 2 .

Procurou-se amostrar sempre no centro da seção de cada ponto utilizando-se um becker de $500 \mathrm{~mL}$, previamente esterilizados. Após a coleta foram avaliados os parâmetros temperatura da água e oxigênio dissolvido. Após término da coleta de dados, os valores foram comparados com as Resoluções n 357 de 2005 e n 430 de 2011 do Conselho Nacional do Meio Ambiente, e foi aplicado o teste de Tukey ao nível de 5\% probabilidade. 


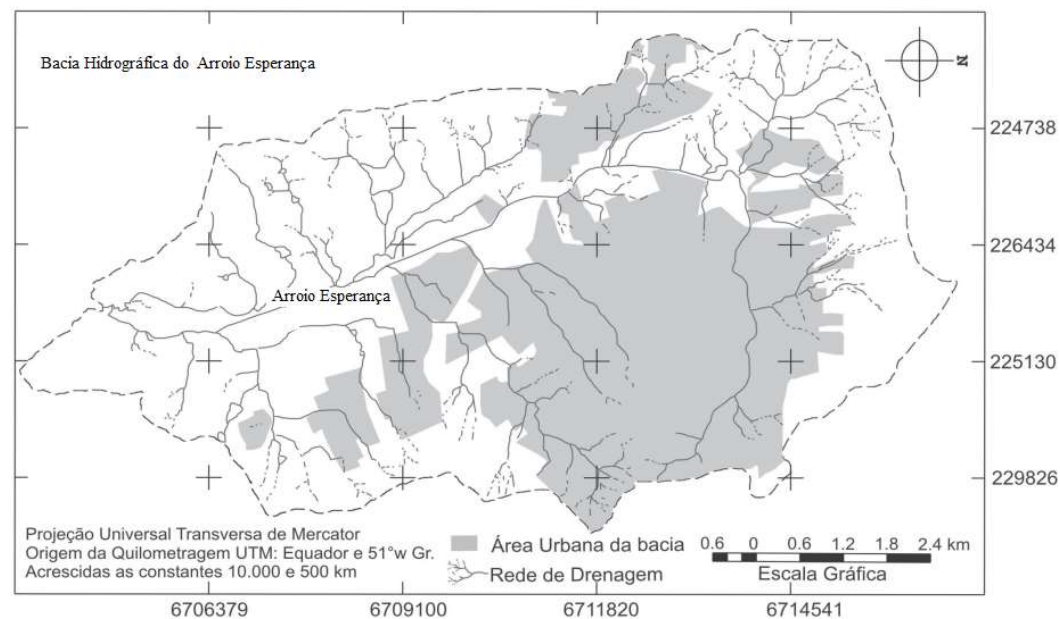

Figura 1 - Mapa de localização da Bacia Hidrográfica do Arroio Esperança em Santa Maria, RS.

\section{RESULTADOS E DISCUSSÃO}

Os resultados das análises da temperatura da água e do oxigênio dissolvido são apresentados na tabela 1 .

Tabela 1 - Média das análises de oxigênio dissolvido (mg/L) e temperatura $\left({ }^{\circ} \mathrm{C}\right)$ nos meses de avaliação, no Arroio Esperança, Santa Maria - RS.

\begin{tabular}{ccc}
\hline Ponto & $\begin{array}{c}\text { Oxigênio Dissolvido } \\
(\mathbf{m g} / \mathrm{L})\end{array}$ & Temperatura $\left({ }^{\circ} \mathbf{C}\right)$ \\
\hline $\mathbf{1}$ & $1,2 \mathrm{~d}^{1}$ & $17,6 \mathrm{c}^{1}$ \\
$\mathbf{2}$ & $1,6 \mathrm{c}$ & $18,0 \mathrm{bc}$ \\
$\mathbf{3}$ & $2,0 \mathrm{~b}$ & $18,4 \mathrm{~b}$ \\
$\mathbf{4}$ & $2,5 \mathrm{a}$ & $19,0 \mathrm{a}$ \\
\hline $\mathbf{C V ~ ( \% )}$ & 21,01 & 3,96 \\
\hline
\end{tabular}

${ }^{1}$ As médias, na vertical, seguidas de mesma não diferem estatisticamente entre si.
No ponto 1, localizado na calha Parshall (Figura 2), observam-se os menores valores de oxigênio dissolvido, com média de $1,2 \mathrm{mg} / \mathrm{L}$, e variação entre $0,61 \mathrm{mg} / \mathrm{L}$ e $2,1 \mathrm{mg} / \mathrm{L}$. Para este ponto, a temperatura média foi de $17,6^{\circ} \mathrm{C}$, variando entre $13,7^{\circ} \mathrm{C}$ e $21,7^{\circ} \mathrm{C}$. A Figura 3 mostra a variação por coleta durante a pesquisa. No ponto 2 , observou-se valores médios de $1,6 \mathrm{mg} / \mathrm{L}$ e $18,0^{\circ} \mathrm{C}$ para oxigênio dissolvido e temperatura, respectivamente. Estes valores variaram entre $0,6 \mathrm{e}$ $2,3 \mathrm{mg} / \mathrm{L}$ para oxigênio dissolvido e 14,2 a $21,4^{\circ} \mathrm{C}$ para a variável temperatura (Figura 4).

O ponto 3 apresentou valor médio de 2,0 $\mathrm{mg} / \mathrm{L}$ para o oxigênio dissolvido, tendo valores situados entre 1,33 e 2,87 mg/L. A temperatura no mesmo ponto apresentou valor médio de $18,4^{\circ} \mathrm{C}$, e variação entre 14,0 e $23,3^{\circ} \mathrm{C}$ (Figura 5). Por último, o ponto 4 apresentou valor médio de 2,5

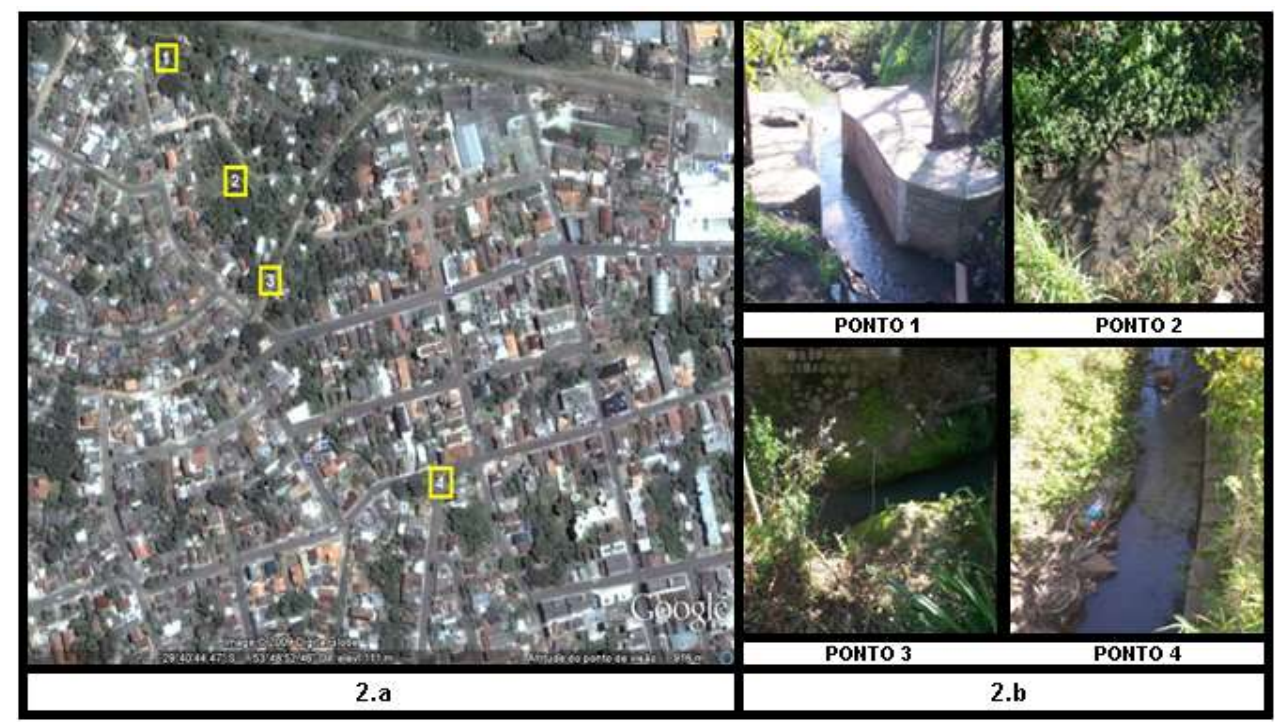

Figura 2 - Localização dos pontos de amostragem via satélite (2.a) e imagem dos quatro pontos de estudo (2.b). 
$\mathrm{mg} / \mathrm{L}$, variando entre 1,64 e 3,94 mg/L. O valor de temperatura da água foi de $19,0^{\circ} \mathrm{C}$ médio, variando entre 15,7 e $23,0^{\circ} \mathrm{C}$ (Figura 6 ).

Estatisticamente, os valores de oxigênio dissolvido variaram entre todos os pontos. Para a variável temperatura, também ocorreu diferença significativa entre os pontos, porém o ponto que diferiu de todos eles foi o ponto quatro, que está situado na cota mais baixa da pesquisa $(96,8 \mathrm{~m})$.

De acordo com a Resolução 357/05 do Conselho Nacional do Meio Ambiente - CONAMA (CONAMA, 2005), os valores de oxigênio dis- solvido estão, considerando os valores médios, fora dos estabelecidos para um rio classe IV para os pontos 1 e 2 , que restringe o seu valor mínimo como 2,0 mg/L e, fora dos estabelecidos para um rio de classe III para os pontos 3 e 4, onde, que restringe o seu valor mínimo como $4 \mathrm{mg} / \mathrm{L}$. Portanto, a água do Arroio Esperança apresenta valores críticos à sobrevivência de formas aeróbicas de vida e indica também a presença de muita matéria orgânica, provavelmente de esgotos domésticos (SPERLING, 1996).

Os valores de temperatura encontrados

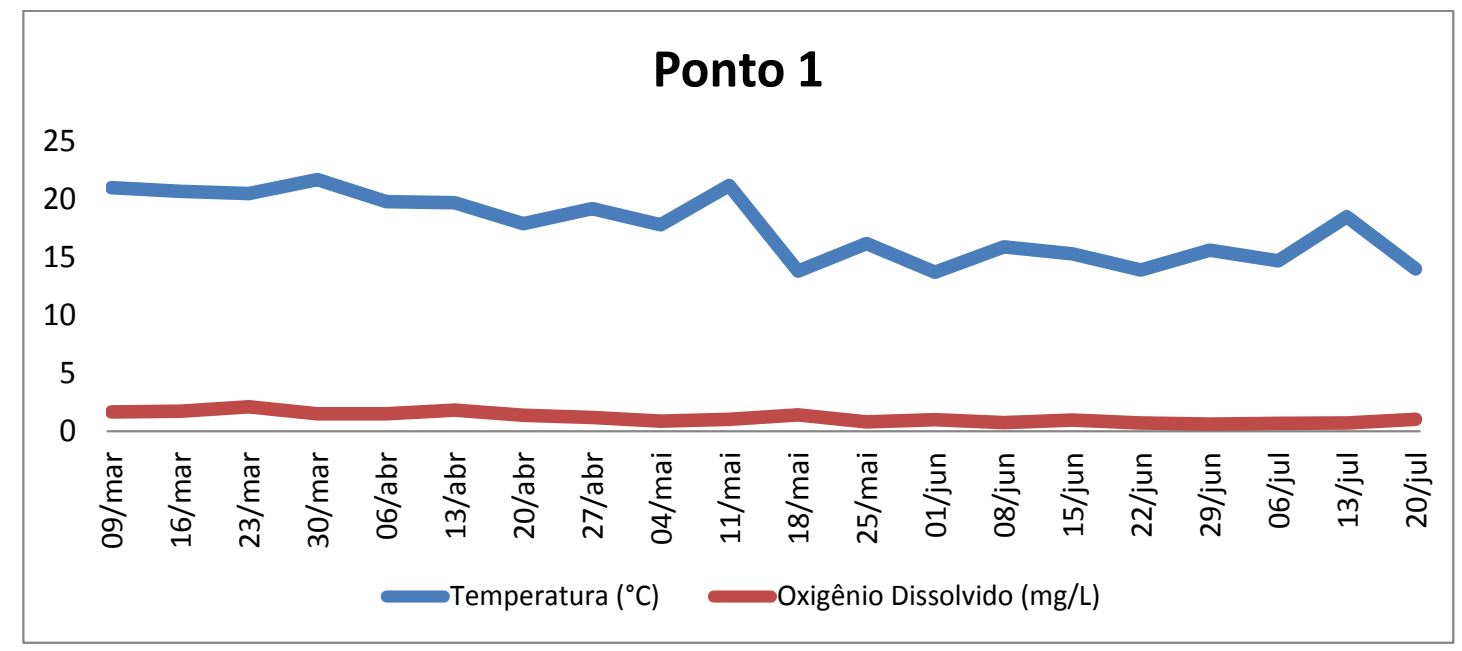

Figura 3 - Variação do oxigênio dissolvido e temperatura no ponto 1 do Arroio Esperança, em Santa Maria - RS.

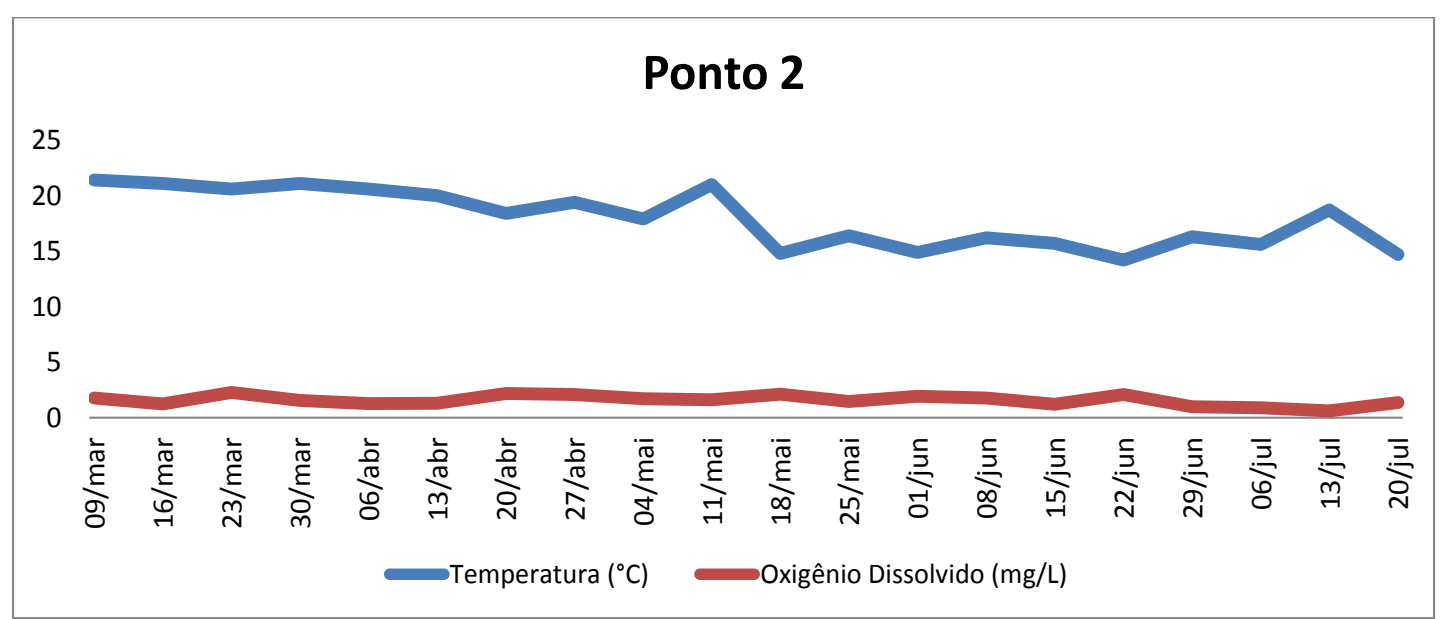

Figura 4 - Variação do oxigênio dissolvido e temperatura no ponto 2 do Arroio Esperança, em Santa Maria - RS. 


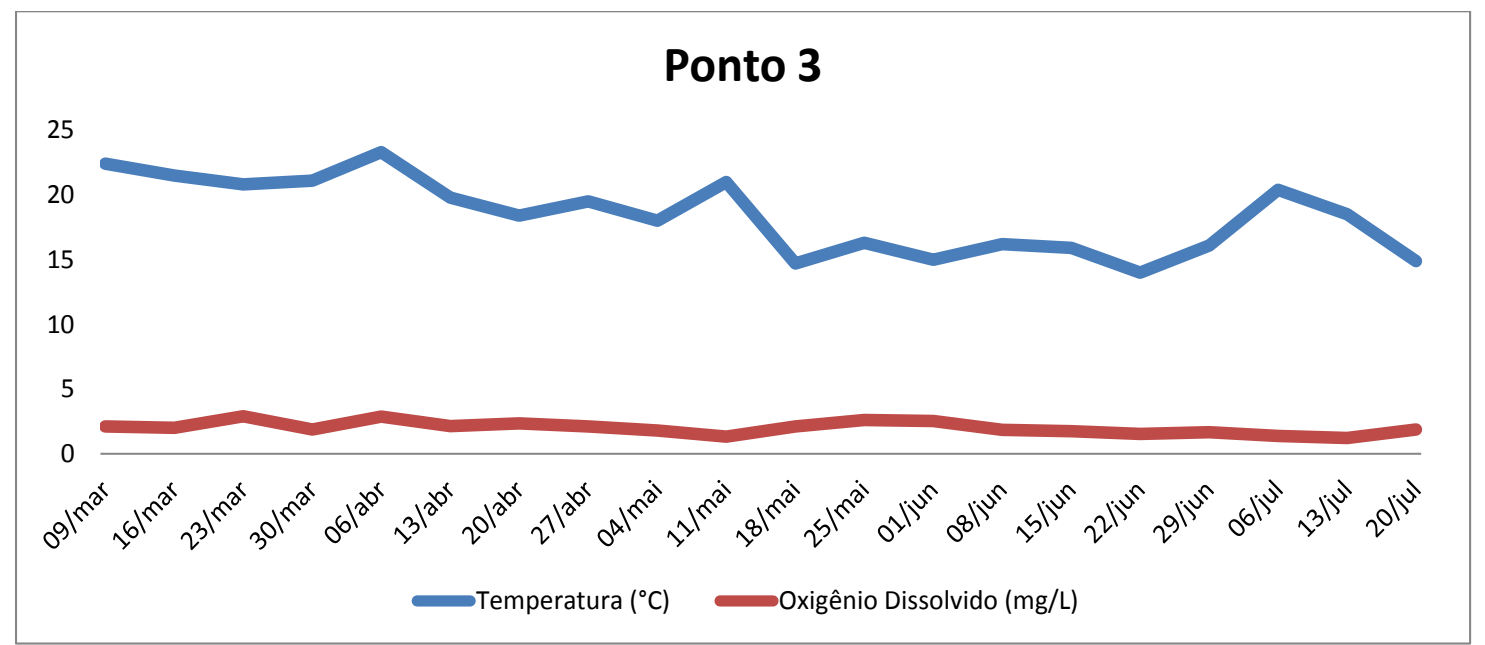

Figura 5 - Variação do oxigênio dissolvido e temperatura no ponto 3 do Arroio Esperança, em Santa Maria - RS.

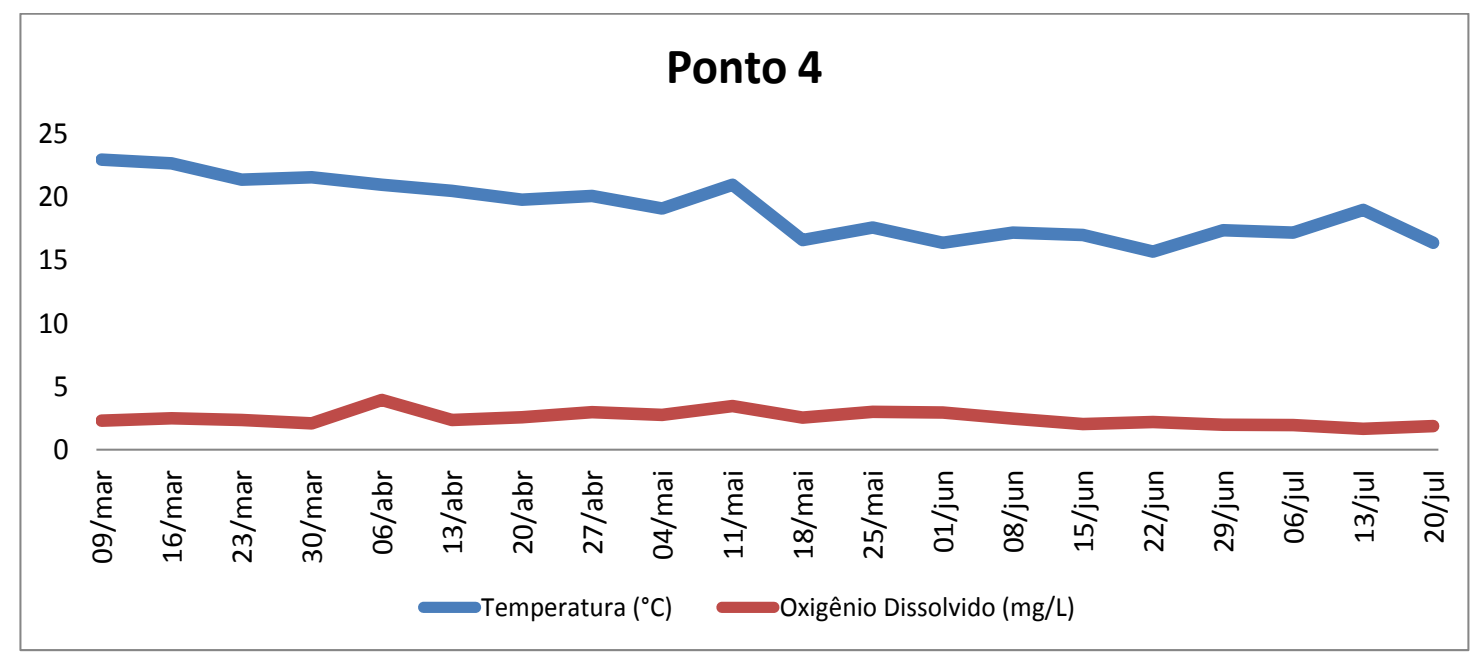

Figura 6 - Variação do oxigênio dissolvido e temperatura no ponto 4 do Arroio Esperança, em Santa Maria - RS.

nesta pesquisa estão de acordo com a Resolução $\mathrm{n}^{\circ} 430$ de 2011 do Conselho Nacional do Meio Ambiente (CONAMA, 2011), que estabelece valor máximo como $40^{\circ} \mathrm{C}$. Nesta pesquisa, os valores não ultrapassaram $23,3^{\circ} \mathrm{C}$ (ponto 3 ).

Observou-se (Tabela 1) que, ao diminuirse a temperatura da água, ocorreu diminuição do oxigênio dissolvido. Segundo Baird (2002), esse fato se justifica porque na queda da temperatura ocorre uma mistura total da massa d'água nas partes superiores até níveis de 10\% de saturação. Esse fenômeno, segundo o autor, pode persistir por até três dias, e é suficiente para provocar intensa mortandade de peixes e causar a redução acentuada da população de fitoplâncton e zooplâncton.

\section{CONCLUSÃO}

Após a análise dos parâmetros oxigênio dissolvido e temperatura da água do Arroio Esperança, chegou-se a conclusão de que fica notória a péssima qualidade deste. Os valores encontrados nesta pesquisa para a variável oxigênio dissolvido mostram que o Arroio está em condições precárias de utilização para quaisquer objetivos. A dinâmica de uso e ocupação do solo de forma desordenada na área da microbacia contribui substancialmente para esta degradação, somando-se às cargas poluidoras de origem doméstica.

\section{REFERÊNCIAS}

ALMEIDA-VAL, V. M. F.; FARIAS, I. P.; SILVA, M. N. P.; DUNCAN, W. P.; VAL, A. L. Biochemical adjustments to hypoxia by Amazon cichlids. Braz J. Med. Biol. Res, v. 28, p. 1257 - 1263. 1993.

ALMEIDA-VAL, V. M. F.; VAL, A. L.; HOCHA- 
CHKA, P. W. Hypoxia tolerance in Amazon fishes: status of na under-explored "goldmine". In: HOCHACHKA, P.W.; LUTZ, P. L.; SICK. T.; ROSENTHAL, M.; VAN DEN THILLART, G. (Ed.). Surviving Hypoxia: Mechanics of control and adaption. Boca Raton: CRC Press, p. 435 - 445. 1995.

wwM.A.L. Recio e L.C.M Carrera. Porto Alegre: Bookman, 2002.

BRANCO, S. M.; PORTO, M. F. A. \& DE LUCA, S. J. Caracterização da qualidade da água. In: PORTO, R. L.L. (Org.) Hidrologia Ambiental. São Paulo: Editora da Universidade de São Paulo: Associação Brasileira de Recursos Hídricos, 1991. p. 27-66

CONAMA - Conselho Nacional do Meio Ambiente (Brasil). Resolução n 357, de 17 de março de 2005. Dispõe sobre a classificação dos corpos de água e diretrizes ambientais para o seu enquadramento, bem como estabelece as condições e padrões de lançamento de efluentes, e dá outras providências.

Diário Oficial [da] República Federativa do Brasil, Brasília, DF, ano 142, n. 53, Seção 1, p. 58-63, 18 mar. 2005.

CONAMA - Conselho Nacional do Meio Ambiente (Brasil). Resolução no 430, de 13 de março de 2011. Dispõe sobre Dispõe sobre as condições e padrões de lançamento de efluentes, complementa e altera a Resolução $\mathrm{n}^{\circ}$ 357, de 17 de março de 2005, do Conselho Nacional do Meio Ambiente-CONAMA. Diário Oficial [da] República Federativa do Brasil, Brasília, DF, n. 92, Seção 1, p. 89, 16 maio 2011.

MERTEN, G. H.; MINELLA, J. P. Qualidade da água em bacias hidrográficas rurais: um desafio atual para a sobrevivência futura. Agroecologia e Desenvolvimento Rural Sustentável. Porto Alegre, v. 3, n.4, out/dez. 2002.

NUVOLARI, A. Esgoto Sanitário: coleta transporte tratamento e reuso agrícola. São Paulo: Edgard Blücher. 2003.

PEREIRA, V.P. Solo: manejo e controle de erosão hídrica. Jaboticabal: FCAV, 1997. 56 p.

SÁNCHEZ-BOTERO, J. I. Distribuição espacial da ictiofauna associada as raízes de macrófitas aquáticas em relação ao oxigênio dissolvido, temperatura e tipo de planta na Amazônia central. Manaus, INPA/FUA. 2000, 47p.
SOARES, M. G. Estratégias respiratórias em peixes do lago Camaleão (Ilha da Marchantaria). Amazônia, INPA/FUA, 1993, $146 \mathrm{p}$.

SPERLING, M. V. Introdução à qualidade das águas e ao tratamento de esgotos. 2 ed. Belo Horizonte, MG: DESA. 1996.

TELLES, D. D. e COSTA, R. H. P. G.. Reuso da água: conceitos, teorias e práticas. 1. Ed. São Paulo: Editora Blucher, 2007.

TUDINSI, J. G. Água no século XXI: enfrentando a escassez. São Carlos, SP: Rima. 2003. 